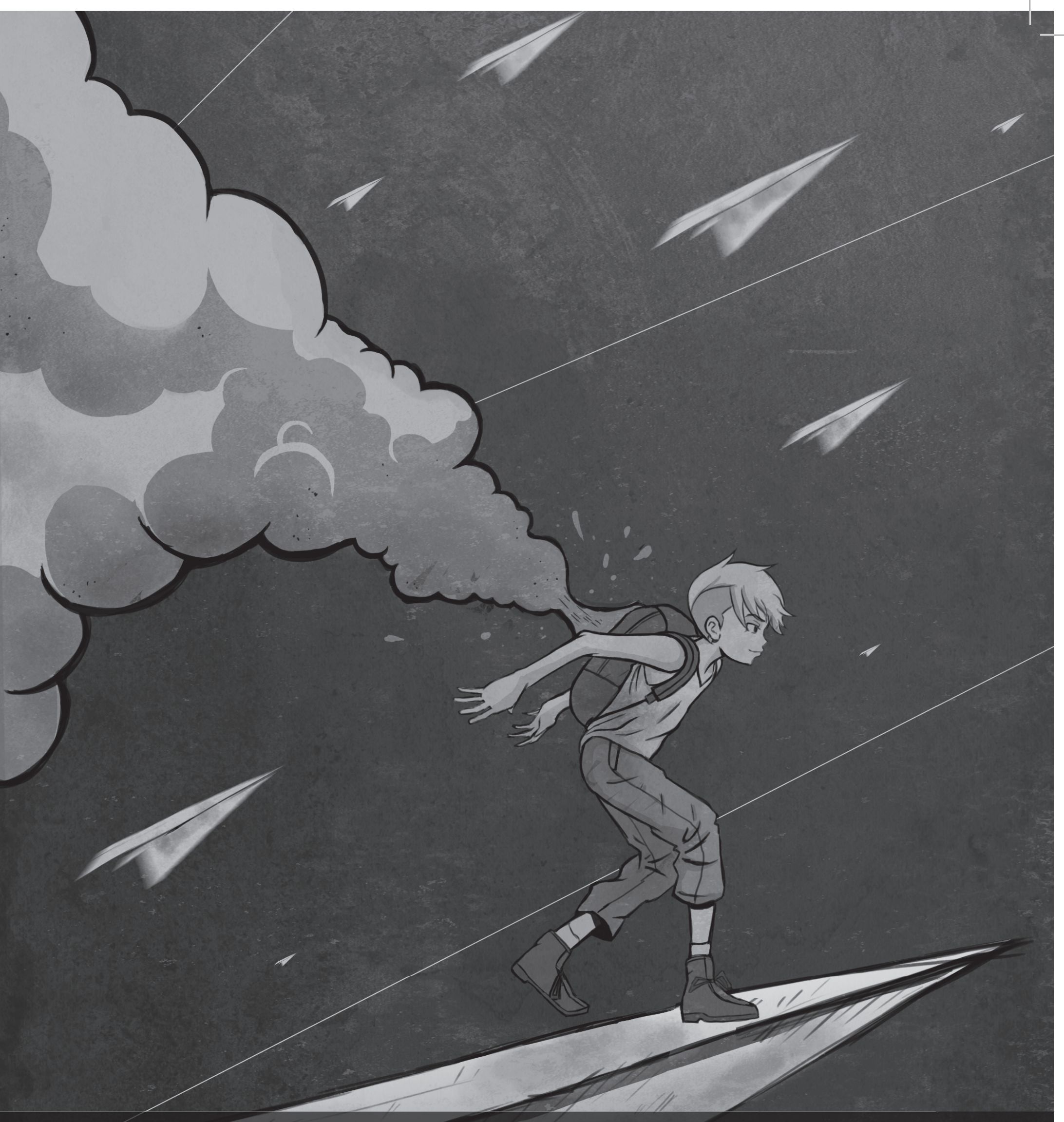

Enfoques de mediación metacognitiva para una docencia universitaria orientada al logro del aprendizaje autónomo 


\section{Enfoques de mediación metacognitiva para una docencia universitaria orientada al logro del aprendizaje autónomo}

Metacognitive mediation approaches for a universitary education geared towards autonomous learning

\author{
Isabel Sierra Pineda (Colombia) \\ Universidad de Córdoba \\ isasierra3@yahoo.com \\ Profesora Titular del Departamento de Informática Educativa de \\ la Universidad de Córdoba-Colombia, desde 1997. Especialista \\ en Computación para la Docencia y Magíster en Educación. \\ Directora Regional, docente e investigadora en el programa de \\ Maestría en Educación del Sistema de Universidades Estatales \\ del Caribe Colombiano (SUE Caribe).
}

\section{Resumen}

\author{
Enfoques de mediação metacognitiva para \\ uma docência universitária orientada ao \\ logro do aprendizado autónomo
}

Este artículo presenta la conceptualización, el diseño y la valoración de un conjunto de estrategias de mediación metacognitiva aplicadas en forma de programa con la finalidad de influir en los procesos de aprendizaje autorregulado y autónomo, lo cual se realizó comparando comportamientos, rasgos y expresiones de autonomía de estudiantes de la licenciatura en informática educativa y medios audiovisuales situados en ambientes convencionales, duales y virtuales, aplicando un diseño cuasicontrol por cohortes, además de una etapa de profundización apoyada en el análisis del discurso. Entre los hallazgos principales, se encontró que en entornos duales (blended-learning), con estrategias de intervención metacognitiva, las características de acceso y disponibilidad de recursos para el estudiante generan condiciones más favorables para la autoeficacia, entendida como confianza en su capacidad de alcanzar resultados. Las consideraciones y reflexiones derivadas de esta investigación que involucró a 81 estudiantes, futuros formadores, se constituyeron en aportes para un modelo innovador de diseño psico-educativo que puede ser implementado en entornos mediados con tecnologías de la información y la comunicación, explícitamente orientados a la generación de autonomía y al ejercicio de la independencia cognitiva.

\section{Abstract}

The following article features the conceptualization, design, and assessment of a set of metacognitive mediation strategies applied as a program with the ultimate objective of influencing the self-regulated and autonomous learning processes. This process was carried out by comparing behaviors, features and autonomy expressions observed in students from the audiovisual media and educative computer science undergraduate program in both dual and virtual conventional environments using a quasi-control design by cohort, complemented by a further study stage supported on discourse analysis.

\section{Resumo}

Este artigo apresenta a conceituação, o desenho e a valoração de um conjunto de estratégias de mediação metacognitiva aplicadas em forma de programa com a finalidade de influir nos processos de aprendizado autorregulado e autônomo, o qual se realizou comparando comportamentos, rasgos e expressões de autonomia de estudantes da licenciatura em informática educativa e meios audiovisuais situados em ambientes convencionais, duais e virtuais, aplicando um desenho quase controle por conjuntos, além do mais de uma etapa de aprofundamento apoiada no análise do discurso. Entre as descobertas 
One of the most remarkable findings was that the characteristics of resources' access and availability for students promote more favorable conditions for self-efficacy, understood as confidence on their ability to meet their objectives. The reflections and considerations derived from this research that engaged 81 students, future trainers, constituted a great contribution to an innovative psycho-educative design model that may be implemented in information and communications technology mediated contexts, explicitly oriented to foster autonomy and cognitive independence exercise.

Palabras claves: aprendizaje autorregulado, mediación metacognitiva, ambientes de aprendizaje, autonomía percibida, autonomía expresada

Palavras chave: aprendizado autorregulado, mediação metacognitiva, ambientes de aprendizado, autonomia percebida, autonomia expressada

\section{Cita recomendada}

Sierra, I. (2012). Enfoques de mediación metacognitiva para una docencia universitaria orientada al logro del aprendizaje. Panorama, 6(11), 65-87. principais, se encontrou que em entornos duais (blended-learning), com estratégias de intervenção metacognitiva, as características de acesso e disponibilidade de recursos para o estudante geram condições mais favoráveis para a autoeficácia, entendida como confiança em sua capacidade de conseguir resultados. As considerações e reflexões derivadas de esta pesquisa que envolveu a 81 estudantes, futuros formadores, se constituíram em aportes para um modelo inovador de desenho psico-educativo que pode ser implementado em entornos mediados com tecnologias da informação e a comunicação, explicitamente orientados à geração de autonomia e ao exercício da independência cognitiva.

Key words: self-regulated learning, metacognitive mediation, learning environments, perceived autonomy, expression of autonomy 


\section{MARCO SITUACIONAL: ¿ES NUESTRA UNIVERSIDAD UNA DE LAS UNIVERSIDADES DEL SIGLO XXI?}

En la Universidad de Córdoba, Colombia, institución pública de educación superior fundada en 1960, como en todas las Universidades colombianas, se implantó el sistema de créditos que orienta la homologación de profesiones para regular y reconocer el tiempo de atención del estudiante a la programación efectiva de docencia directa, y su dedicación a las tareas realizadas en forma independiente.

La Universidad de Córdoba tiene cinco facultades y una oferta académica de 26 programas de formación profesional en pregrado y 15 en postgrado.

Desde la Facultad de Educación y Ciencias Humanas, se ofrecen siete programas de formación de licenciados, con el fin de convertirlos en profesionales de la educación en diferentes áreas y especificidades. Entre el profesorado de esta facultad, el sistema de créditos ha sido motivo de algunos debates desde hace varios años, y se reconoce al menos teóricamente, que la adopción de este sistema plantea una serie de implicaciones pedagógicas y la necesidad de implementar una mudanza en las concepciones de enseñar y de aprender, ya que en la actualidad la enseñanza se entiende como un proceso de mediación y el aprendizaje como un proceso autónomo y permanente, basado en la autorregulación de la actividad cognoscitiva. Ambos procesos le exigen tanto a alumnos como a profesores capacidades y habilidades de organización..

Por eso el rol del enseñante, nunca como ahora, en las denominadas sociedades del aprendizaje, necesita hacer una transición verdadera hacia el papel de mediador para que no solo organice los contenidos a aprender, sino que disponga estructuras adecuadas de andamiaje, mediante actividades y recursos diseñados adecuadamente para que el estudiante sea quien elabore y construya representaciones, a partir de contenidos para que finalmente aprenda.

Se requiere además que el docente asuma la tutoría como una función de acompañamiento, monitoreo del trabajo independiente y de evaluación formativa para darse cuenta del progreso del estudiante y, así, orientarlo gradualmente hacia una independencia cognoscitiva, basada en el ejercicio de competencias. Estos roles sin duda le implican a los profesores, en principio, no solo mayor tiempo y cuidado en la preparación de las estrategias y actividades sino mayor tiempo de dedicación individual hacia los estudiantes; porque es necesario que los alumnos mejoren su conciencia para usar sus capacidades y recursos cognitivos, adoptar estrategias de aprendizaje, de autoevaluación que les permitan la interpretación de contenidos, la planificación de su acción sobre los mismos y la elaboración para gestionar aprendizajes duraderos, significativos y eficaces, es decir, asumir la tarea de aprender a pensar y a aprender con autonomía intelectual y ética.

Con el proceso de integración de recursos tecnológicos de tipo telemático, servicios y herramientas de comunicación y organización en la web, el profesorado de la Facultad de Educación y Ciencias humanas de la Universidad de Córdoba-Colombia, que interactúa con alumnos en entornos virtuales, se dedica gran parte del 
tiempo a aprender a usar de manera efectiva las herramientas de las plataformas y algunos de los obstáculos que debe vencer diluyen los objetivos de su actuación interventora o mediadora del aprendizaje.

Entre estos obstáculos, se encuentra la dificultad de crear (digitalizando gran volumen de textos o documentos para colgarlos o subirlos en la plataforma web) o hallar y seleccionar el material digital adecuado y contextualizarlo. Además, la gestión del gran número de comunicaciones de los alumnos, lo pueden alejar del sentido creativo y propositivo de encontrar y aplicar estrategias y actividades enriquecedoras más propicias, para guiar y educar en la reflexión, llevar a la independencia en la gestión de información y fortalecer la construcción de conocimiento, y, desde allí, lograr la autonomía.

Las transiciones no han sido fáciles. En la formación universitaria se mantiene la tensión histórica entre la dirección del diseño instruccional más tradicional y las prácticas abiertas de aprendizaje vivencial en camino al constructivismo. Por ello, se ha enfatizado el desarrollo de competencias asociadas con el dominio de contenidos disciplinares. Desde las decisiones metodológicas del profesorado universitario, tradicionalmente se ha prestado menos atención a la responsabilidad de desarrollar las capacidades de aprendizaje de los estudiantes, a la conciencia sobre su conocimiento y al uso reflexivo y estratégico de sus instrumentos intelectuales.

Se piensa todavía la universidad como fuente de saber y se reconoce un poco menos como un escenario para aprender. Así en la Universidad de Córdoba, se encuentra con demasiada frecuencia que lo único que ha cambiado son los nombres de los cursos y su intensidad horaria programada pero en esencia "la clase" es el único espacio de contacto profesor-alumno y de despliegue de contenidos-actividades; la constante sigue siendo la heteroregulación del aprendizaje, pues se conserva la estructura de interacción y de manejo del tiempo tal como tradicionalmente se ha concebido en educación básica, media y superior; y, en muchos casos, en la cotidianidad del desarrollo del plan de estudios, porque se reduce el sistema de créditos al formalismo de los documentos revisados por el Ministerio de Educación para la aprobación de los programas. La supuesta transición metodológica requerida en la docencia y en el manejo del tiempo independiente del estudiante escasamente trasciende lo conceptual. Quienes actualmente nos responsabilizamos de cursos universitarios, tenemos a nuestro alcance mucha literatura de referencia sobre el deber ser de la universidad y sobre los análisis que se han hecho alrededor de los cambios que se deben operar en las estructuras y en las funciones de la educación superior, afectando las visiones, concepciones y actuaciones de estudiantes y profesores.

Los avances teóricos de la psicología cognitiva y las aportaciones de enfoques constructivistas han contribuído a nuevos modelos de comprensión del aprendizaje universitario y han iluminado el diseño de formas de aproximación metodológica distintas en la didáctica universitaria.

La flexibilización de las instituciones de educación superior implica un proceso de adaptación a las necesidades de la sociedad actual y entre los requisitos de esa transformación se sustenta el papel de las tecnologías de la información y la comunicación como mediatizadoras en los procesos de formación. Esa flexibilización significa revaluación de concepciones, de actitudes, roles y responsabilidades en los estudiantes, en los profesores y en la 
organización propiamente dicha, requiriéndose formas distintas de diseño educativo, de despliegue de contenidos, de la actividad didáctica y de la evaluación de la calidad del aprendizaje de los estudiantes.

Está claro que hoy no debería representar un problema mayor, la falta de bibliografía o la dificultad de acceso a las fuentes de información porque aún en las localidades marginales, los aprendices universitarios están constituyendo prácticas que les permiten hacer contacto con contenidos de diferente grado de elaboración y profundidad con pares y organizaciones a través de la web. Se ha observado también que en la mayoría de los casos y, de manera intuitiva, los estudiantes van generando itinerarios personales de navegación, configurando hábitos y estilos (no reflexionados) de búsqueda, gestión y reorganización de lo que leen en las autopistas telemáticas o de lo que descubren en los repositorios de objetos digitales. Ello les permite pasar de una representación a otra, reciclando, parafraseando y, en el mejor de los casos, aplicando conceptos para resolver las tareas que se les proponen.

Entre las dificultades que se señalan con frecuencia, en relación con el aprendizaje en estudiantes universitarios, están las deudas que tienen con los niveles de formación precedentes, una baja comprensión de las implicaciones del sistema de créditos académicos, en relación con la metodología de los docentes y con las prácticas de estudio de los estudiantes, y una deficiente estimulación por el aprendizaje autónomo. Así, el aumento de la accesibilidad a fuentes de información no trae necesariamente aparejado una mejora en sus habilidades de pensamiento crítico-propositivo, ni en la consolidación de enfoques de aprendizaje profundo, características que se deberían evidenciar en este nivel de formación.

A partir de lo anterior, el eje propiciador de esta experiencia docente-investigativa fue el interés por comprobar, documentar y sustentar los cambios ocurridos en los métodos de enseñanza, que aunque son requeridos, no han ocurrido, en la ineludible transición del trabajo de ambientes de aula "cara a cara", a la formación apoyada con recursos mediáticos en la Facultad de Educación en la Universidad de Córdoba - Colombia, específicamente en La licenciatura en informática y medios audiovisuales.

Los entornos virtuales son actualmente ambientes hipermedia caracterizados no solo como espacios para uso de herramientas y recursos, sino como escenarios experienciales enriquecidos de estudio-trabajo, espacios para el análisis de contenidos, para la búsqueda de la comprensión, la gestión del conocimiento útil y la aplicación del mismo en contexto. Nuestros aprendices entonces requieren de una gama de habilidades, estrategias y competencias que le permitan poner estos ambientes al servicio de sus procesos de aprendizaje. Pero, ¿de qué tipo de aprendizaje se habla?

En el debate propuesto sobre esta problemática que se ha suscitado también a nivel regional en el Caribe colombiano, acerca de las características del aprendizaje universitario en un contexto cada vez más penetrado por tecnologías de información y comunicación, se pretende aportar construcciones a partir de la experiencia vivida en la Facultad de Educación de la Universidad de Córdoba, en el marco de una investigación realizada con diseño cuasiexperimental con grupos control por cohortes y con estudiantes de sexto semestre en el contexto de sus clases en la licenciatura en la Facultad de Educación de la Universidad de Córdoba. Con el propósito de establecer 
la relación entre las estrategias de enseñanza metacognitivas en ambientes virtuales y los niveles de desarrollo de la autorregulación y aprendizaje autónomo se han elaborado una serie de constructos sobre la influencia que tienen las acciones docentes trabajadas con este enfoque de mediación social cognitivo en el aprendizaje de los estudiantes universitarios, en la valoración y evaluación de los resultados de su trabajo académico y la percepción que estos tienen sobre sí mismos, sobre sus habilidades, sobre el conocimiento y sobre la responsabilidad de seguir aprendiendo de manera independiente. La experiencia permitió analizar las diferencias entre las dinámicas y percepciones que se generan en los estudiantes situados en ambientes de docencia presencial convencional, y los que desarrollan su actividad académica en ambientes mediados tecnológicamente, bien apoyados en entornos duales o híbridos (blended learning) o de manera completamente virtual. Se contrastaron las interacciones y los efectos de la intervención didáctica diseñada con enfoques metacognitivos desde la perspectiva del paradigma mediador (Feuerstein, 1979; Tébar, 2003) en los procesos de desarrollo del aprendizaje autónomo y autorregulación en estudiantes universitarios.

\section{Premisas psicoeducativas sobre la metacognicion, la autorregulación del aprendizaje y la autonomía}

Teniendo en cuenta que uno de los fines de la educación superior en Colombia es lograr que los estudiantes desarrollen una independencia intelectual y ejerzan plenamente la autonomía, y considerando que la base de esa autonomía está en la capacidad metacognitiva, uno de los temas importantes en educación es cómo promover el aprendizaje autónomo, sus factores y variables asociados con su expresión desde estrategias de enseñanza explícitas y conscientemente diseñadas para ese fin usando las mediaciones disponibles y asumiendo roles apropiados en consistencia con los ambientes que hoy se proponen como alternos para los procesos de formación.

Así, cuando se proponen programas de formación en modalidad virtual, a distancia y e-learning o cuando se integran las formas de enseñanza convencional presencial con entornos mediados por tecnologías web, una de las características deseables, y el principal propósito del profesor- tutor y del entorno mediador, debe estar enfocado en favorecer el ejercicio de la autonomía y que los estudiantes logren desarrollarla.

Por ello, se estudiaron -como base para dar sustento a la intervención -las variables y componentes de la metacognición; como fenómeno humano, la autorregulación como proceso psicológico complejo; y el aprendizaje autónomo como fin deseable en la formación de la educación superior. Se documentaron los orígenes, la evolución y las relaciones entre estos constructos y se indagó sobre las implicaciones de los ambientes virtuales en educación superior, centrándose en las nuevas demandas del aprendizaje universitario, los perfiles y actitudes de educandos y docentes, y los requerimientos de calidad pedagógica en estos entornos mediados por tecnologías web.

Los estudios de metacognición (Flavell \& Wellman, 1977) se consideran fundamentales en la sustentación de una pedagogía del aprendizaje autónomo, y se pueden establecer variados contrastes acerca de este concepto, considerado como un constructo tridimensional que implica que el individuo conozca su propio proceso de aprendizaje, programe conscientemente sus estrategias de aprendizaje, de memoria, de solución de problemas y toma de decisiones, evalúe sus reacciones y controle la capacidad de transferir esas estrategias y habilidades a diferentes situaciones o contextos. 
Por ello según planteamientos de Boekaerts y Niemivirta (2000), activar los procesos de autorregulación implica el uso de estrategias metacognitivas, además del esfuerzo y la motivación en los aprendices, todo ello en función de las condiciones de los contextos en que están situados los grupos de estudiantes. Desde la acción de los educadores, para enseñar a los alumnos a aprender requiere una pedagogía con abordaje metacognitivo: poner especial énfasis en los procesos de interiorización: orientar en los aprendices el pensamiento sobre sí mismos, pero también orientar a la conciencia.

\section{Figura 1. ElEMENTOS CONSIDERADOS PARA LA INDAGACIÓN TEÓRICA COMO SUSTENTO DE LA INVESTIGACIÓN}

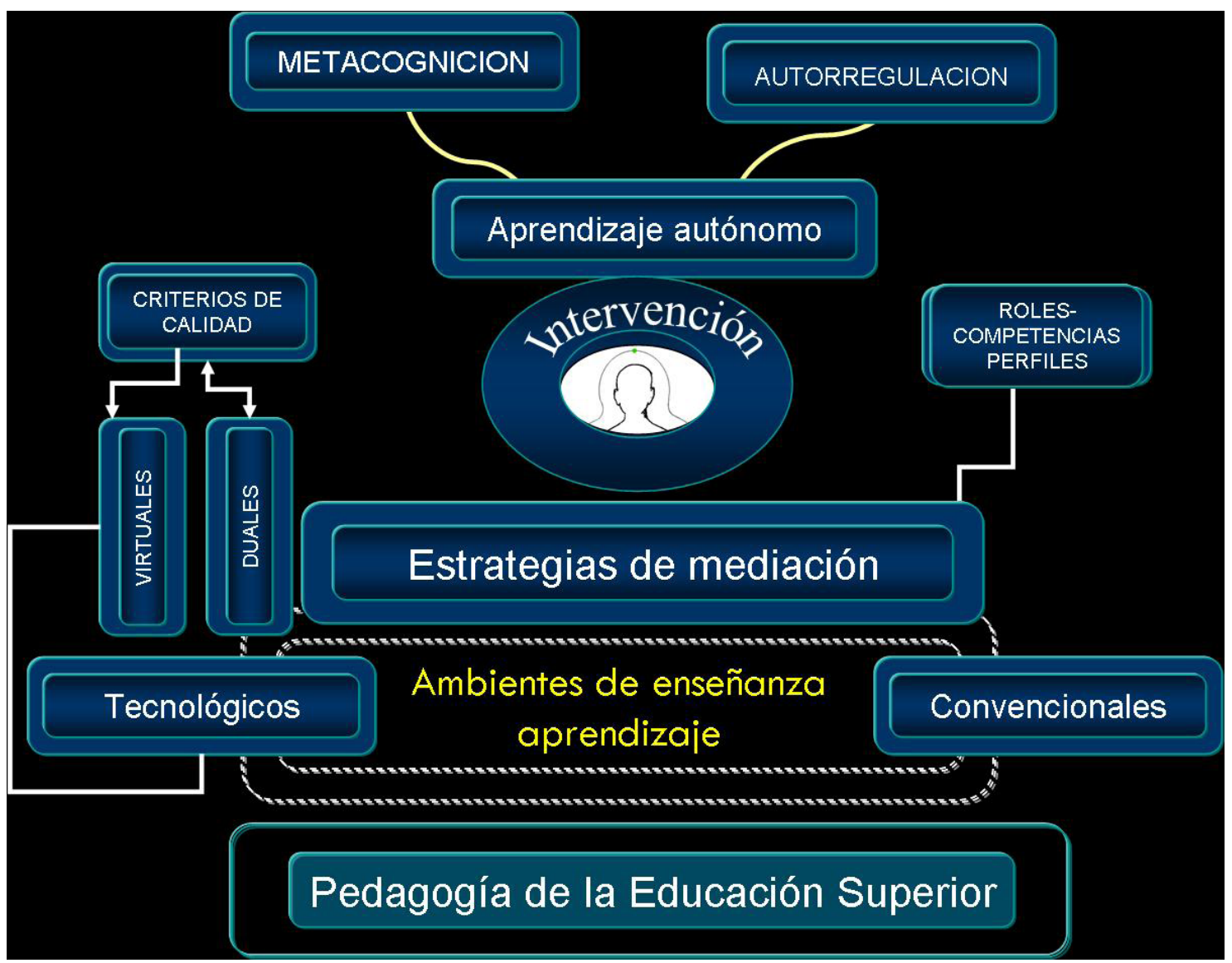

Fuente: Elaboración propia

La base para configurar la propuesta de intervención con enfoque de mediación metacognitiva, que se constituyó en el tratamiento para los grupos experimentales, estuvo basada en tres tipos de referentes: 1) principios sobre la pedagogía de la mediación y las estrategias de aprendizaje mediado (Tébar, L. 2003 Feuerstein, 1980); 2) Principios teóricos, hallazgos y conclusiones de estudios empíricos previos de la enseñanza metacognitiva y programas para el desarrollo de la autorregulación del aprendizaje (Zimmerman, 2001; Boekaerts, 2000); y 3) 
constructos derivados del análisis de resultados de las encuestas a docentes y de la síntesis de pautas metacognitivas propuestas por docentes de la Facultad de Educación de la Universidad de Córdoba.

El referente de las Estrategias de Aprendizaje Mediado (EAM), (Feuerstein, 1980), y de la Evaluación Dinámica Mediada, (EDM), representó para este estudio una fuente de ideas, conceptos y metodologías para la formulación de constructos en la articulación de una estrategia integral de intervención apoyada tal como hemos visto en su característica, en la interrogación por etapas y en la inducción a la autointerrogación; de manera que en un formato semiestructurado, y a la vez flexible en el contexto de su aplicación, se induzca al estudiante universitario en un ambiente de trabajo que lo motive a alcanzar independencia cognoscitiva, a partir de mejores procesos metacognitivos y de autorregulación de su aprendizaje.

\section{Desarrollo de la experiencia docente-investigativa: el diseño y aplicación de estrategias de media- cion metacognitiva}

El estudio empírico se orientó hacia un objetivo principal: el de verificar mediante un análisis comparativo intragrupo e intergrupos, la presencia de diferencias significativas en el desarrollo de procesos de autorregulación del aprendizaje de los estudiantes situados en ambientes virtuales, ambientes duales mediados con tecnologías de la información y comunicación, y ambientes convencionales de clase cuando hay intervención con estrategias de mediación metacognitiva y cuando no hay este tipo de intervención. Se definió como un diseño cuasi-control por cohortes y se desarrolló durante los semestres académicos desde el año 2007-2008, con 81 estudiantes en tres grupos control y tres grupos experimentales. Estos estudiantes de tercer año (sexto semestre) del curso de Cognición y computación de la Licenciatura en Informática y Medios audiovisuales de la Facultad de Educación en la Universidad de Córdoba-Colombia establecieron sus percepciones sobre sí mismos, especialmente en cuanto al aprendizaje de sus procesos metacognitivos, su autorregulación y su propia autonomía, en los ambientes intervenidos.

Los grupos se definieron y organizaron así:

Grupo 1: Situado en ambiente de clase convencional/ con tareas convencionales para su tiempo de trabajo independiente.

Grupo 2 : Situado en ambiente virtual modalidad dual/ con tareas convencionales para su tiempo de trabajo independiente.

Grupo 3: Situado en ambiente virtual / con tareas convencionales para su tiempo de trabajo independiente.

Grupo 4: Situado en ambiente de clase convencional/ con Tareas metacognitivas para su tiempo de trabajo independiente.

Grupo 5: Situado en ambiente virtual modalidad dual/ con tareas metacognitivas para su tiempo de trabajo independiente.

Grupo 6: Situado en ambiente virtual/ con tareas metacognitivas para su tiempo de trabajo independiente.

El recorrido metodológico, se constituyó por dos miradas complementarias: una, analítica a partir de los datos cuantitativos derivados de la pre y post prueba, y una más interpretativa a partir de la información cualitativa expresada en el discurso de los sujetos experimentales. Se consideró como variable independiente las estrategias 
de mediación metacognitiva expresadas en el programa de intervención; y como variable moderadora el tipo de ambiente de aprendizaje (entorno web virtual y dual); y finalmente como variable dependiente: la autorregulación académica, entendida como regulación de la cognición y del aprendizaje. Se utilizaron como instrumentos pre y post de intervención la escala de autoinforme EDAOM, el cuestionario de Estilos de Aprendizaje, y la autorregulación y orientación motivacional (Castañeda, S.,1999).

TABLA 1. Sistema DE VARIABLES PARA ESTABLECER RELACIONES ENTRE MEDIACIÓN Y AUTORREGULACIÓN

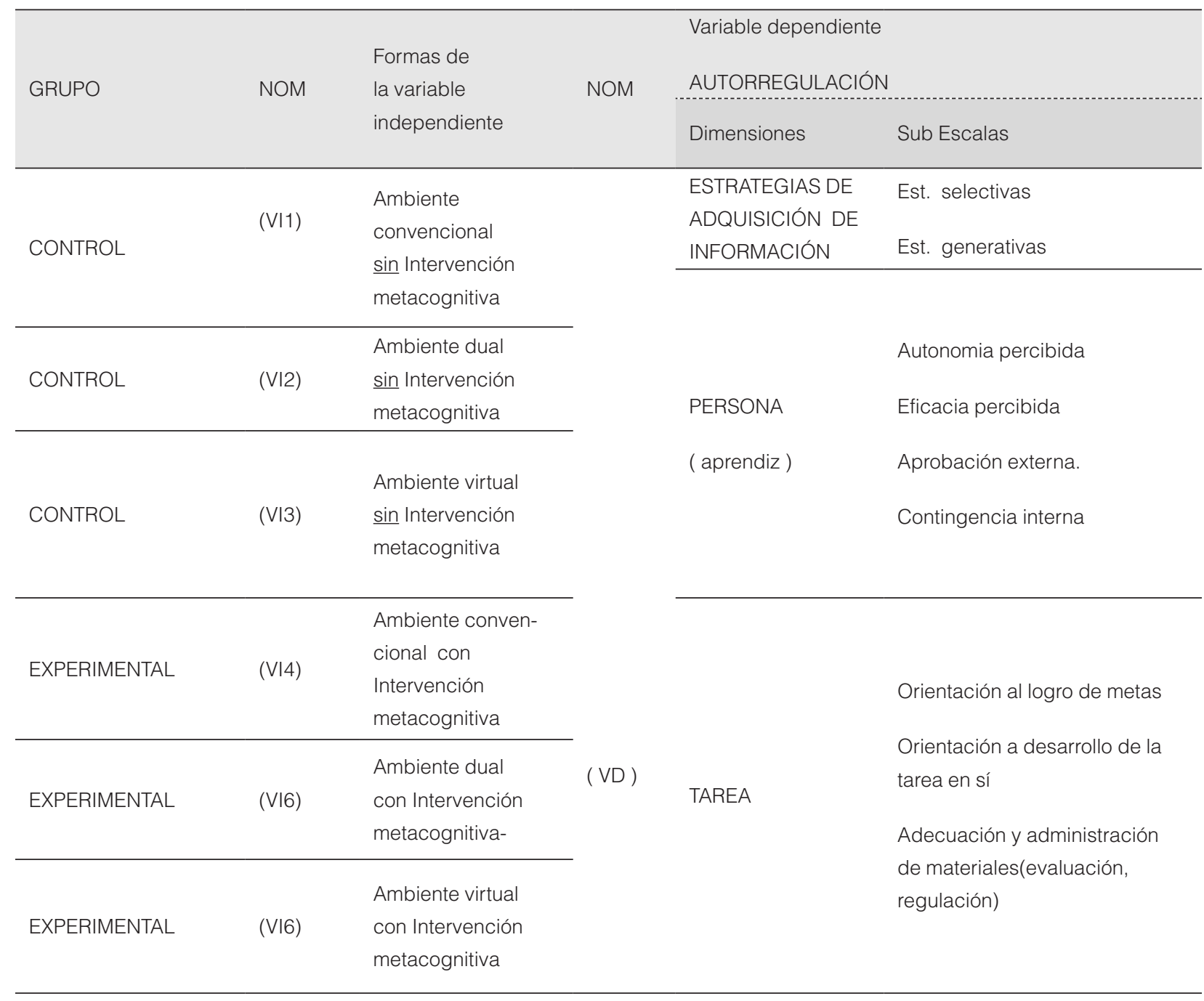

Fuente: Elaboración propia

La intervención se configuró a través del, Programa de Intervención con Estrategias de Mediación Metacognitiva (PIEM). El ambiente mediado metacognitivamente se diseñó y se organizó basado en los criterios desde el estudio de Sierra, 2006 (Entornos virtuales y duales con orientación metacognitiva), para que el estudiante 
pudiera utilizar: 1) los recursos en la búsqueda de información y la gestión, y en el envío de tareas; 2) enlaces a otros portales, 3) referencias; 4) herramientas de diseño de mapas conceptuales; 5) bitácoras o diarios; 6) herramientas de transferencia de archivos, 7) blogs o editores colaborativos; y 8) espacios mediadores de reflexión, comunicación, escritura autoevaluación e interacción.

Para estos estudiantes experimentales de los grupos virtual y dual, se dispuso un acceso identificado al portal, en donde se organizaron los contenidos y recursos, actividades y guías de mediación con enfoque metacognitivo, usando las herramientas de la plataforma Moodle/Genius que le permite al profesor la gestión, administración y fácil actualización de contenidos y supervisión de actividades de usuarios; y le facilita a los estudiantes registrados, escenarios sencillos y transparentes para la realización de actividades, entrega de resultados de tareas, resolución de cuestionarios y el desarrollo de la interacción y edición individual y colaborativa de contenidos.

\section{Fases previas a la intervención metacognitiva}

Inducción del programa de intervención con estrategias de mediación metacognitiva. Se realizó la inducción a los estudiantes en los distintos grupos sobre la estructura del contenido del curso, la metodología, loscriterios de evaluación y la dinámica a desarrollar para cada caso. La inducción para los estudiantes que usarían el entorno apoyado en la web incluyó una familiarización con los aspectos instrumentales y de afianzamiento en el uso de las herramientas de la plataforma gestionada para ese fin bajo un entorno Moodle en el sitio www.edunexos.edu.co.

Desarrollo del programa de intervención con estrategias de mediación metacognitiva. Se prepararon los materiales y se articularon los contenidos, el sistema de tareas, recursos y mediaciones de apoyo y el calendario fue presentado gradualmente durante el semestre como guías en forma impresa a los estudiantes del grupo experimental convencional; se desarrollaron las actividades, se realizaron evaluaciones y se apoyó de manera tutorial asincrónica a los estudiantes de los grupos experimental dual y experimental virtual.

El ambiente del curso en el portal de edunexos/genius, se organizó de manera que quedara disponible en línea para los grupos experimentales, duales y virtuales, durante la fase de tratamiento que se cumplió durante 16 semanas del período académico 1-2008. Durante esta fase experimental se desplegaron los contenidos del curso, se propusieron las tareas y se desarrolló la interacción.

- Implementación. El programa de intervención con estrategias de mediación metacognitivas se desarrolló en tres fases: Premediación, (o inicial), Mediación, (Intervención propiamente dicha) y Postmediación (o de soporte).

La intervención se definió a través de cinco elementos: 1) contenidos, 2) instrucciones - autoinstrucciones, 3) actividades, 4) interrogaciones, y 5) orientaciones situadas u organizadas dentro de las siguientes dimensiones: 
a. EMCC elemento referido a conocimientos, contenidos o conceptos

b. EMCE elemento referido a estrategias

c. EMCT elemento referido a tareas

d. EMCP elemento referido a condiciones personales

e. EMCM elemento referido a materiales o recursos

Las estrategias de mediación metacognitiva, (EMCC,EMCE, EMCT, EMCP, EMCM) aparecen como guías de orientación e instrucción metacognitiva con las que se promueve la auto-instrucción, y se modela la autointerrogación. Se aplicaron cuestionarios de reflexión, independientes del contenido, se entregaron formatos para el desarrollo de talleres, tareas y proyectos, así como los criterios para presentación de trabajos semestrales. Se evaluaron las siguientes subescalas: contingencia interna, aprobación externa, autonomía percibida, eficacia percibida, y administración de recursos o materiales variables, orientación al logro, orientación a la tarea en sí, estrategias generativas, estrategias selectivas de procesamiento de información.

\section{Figura 2. Estructura y PROCESOS DEL PROGRAMA DE INTERVENCIÓN CON ESTRATEGias de MedI-} ACIÓN METACOGNITIVA (PIEM)

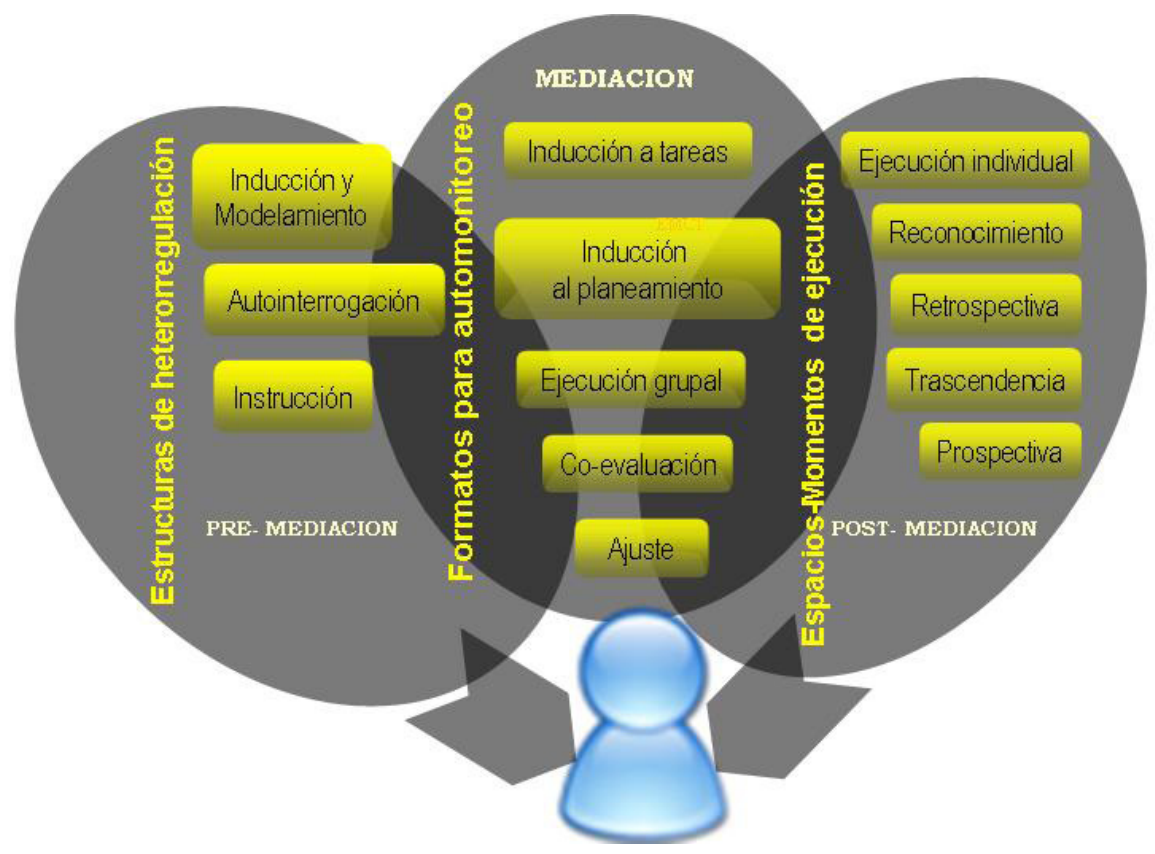

Finalizada la etapa de intervención se realizaron los procesos de análisis de la información. Usando el lenguaje R, se aplicó un entorno para análisis estadístico y a partir de la mediana de los puntajes y porcentajes obtenidos en cada subescala EDAOM, como medida de referencia.

Se realizó un análisis de Kruskal Wallis, una prueba Wilcoxon y una prueba de U de Mann Whitney para determinar la equivalencia entre grupos al inicio y la presencia de diferencias significativas entre grupos de datos 
obtenidos de muestras independientes y muestras relacionadas en los diferentes ambientes, grupos control y experimentales. La intención fue determinar la dependencia o interacción entre tipo de ambiente (Factor Grupo - Tipo de ambiente) y estrategia metacognitiva (Factor Tratamiento-Ausencia-presencia de PIEM), y su influencia en procesos de autorregulación del aprendizaje.

\section{Tabla 2. Pruebas comparativas pre y post de muestras Relacionadas E INDEPENDIENTES}

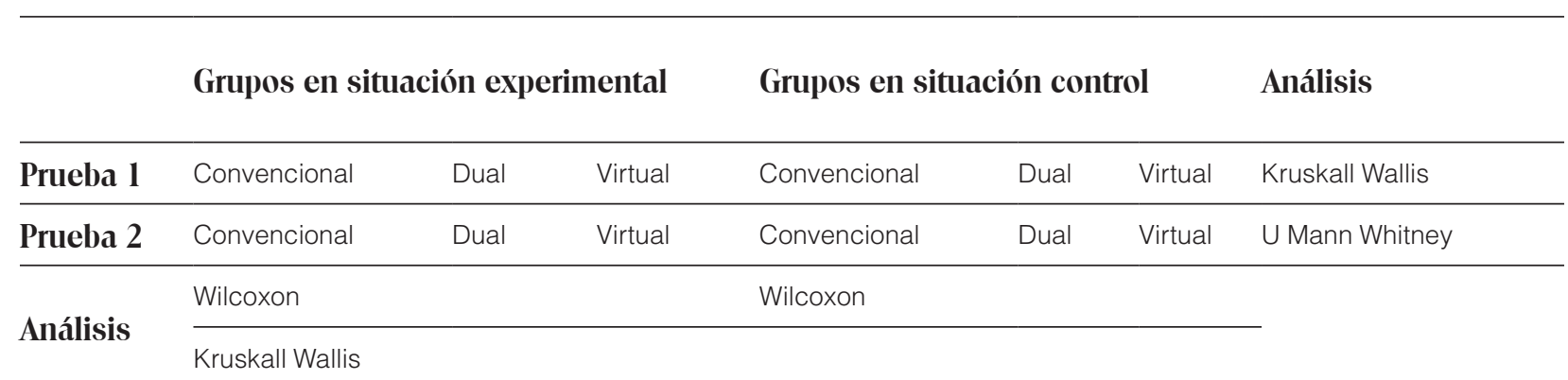

Además con fines interpretativos y de complementación se realizó un análisis de discurso usando el software Atlas Ti, para establecer la presencia sincrónica y diacrónica de rasgos de autonomía expresada en 21 relatos de autoevaluación de una submuestra de estudiantes de los grupos experimentales del estudio. Aquí se definieron dos categorías de análisis a priori: 1) percepción de comportamiento autorregulado y 2) conciencia metacognitiva.

\section{HALLAZGOS Y APRENDIZAJE A PARTIR DE LA EXPERIENCIA DE IMPLEMENTACION DEL PIEM}

Los resultados del análisis estadístico indican que si bien hay diferencias significativas en autorregulación en los tres grupos experimentales, (convencional, dual y virtual intervenidos con el PIEM), en los indicadores de las escalas: autonomía percibida, contingencia interna y eficacia percibida, la evidencia no es suficiente para asegurar que se puedan atribuir estas diferencias únicamente a la intervención con estrategias de mediación metacognitiva.

Tampoco es posible afirmar que en los entornos o ambientes apoyados en recursos virtuales haya una mejora sustantiva de los comportamientos autorregulados o que se genere motivación intrínseca u orientación a la realización autónoma por el solo hecho de contar con herramientas enriquecidas, dado que hubo también mejoras en algunas escalas en algunos de los grupos control. 
Tabla 3. Guía para el anÁlisis del discurso - Sistema de Categorías

\begin{tabular}{ll}
\hline MACROCATEGORÍA & CATEGORÍAS \\
\hline Conciencia Metacognitiva & Conocimiento consciente del contenido visto y de los procesos desarrollados y la capa- \\
& cidad para aplicarlos. \\
& Habilidad consciente para usar las estratategias de manera planificada. \\
Autonomía & Capacidad de evaluar lo que se hace y el significado de lo que se hace, pensar sobre sí \\
Expresada & (Flavell y Wellman 1977; Baker y Brown, 1984; Weinstein, 1985; Bornas, 1994; Pintrich, 1986). \\
Evidencias de & Percepciones de Autoregulación \\
funcionamiento metacognitivo & Conceptos enunciados sobre la propia capacidad estratégica en contexto. \\
y autorregulado & Enunciados sobre las transformaciones de motivación extrínseca a intrínseca. \\
Auto-observación de la conducta, reconocimiento de autorreacción.- \\
Comparación y ajuste de niveles de ejecución basado_en criterios. \\
(Zimmerman; 1994, Brocket e Hiemstra ; 1993; Monereo y Castelló,2001).
\end{tabular}

Figura 3. EXPRESIONES EN SUJETOS EXPERIMENTALES GRUPO VIRTUAL, ANALIZADAS CON AtLAS TI

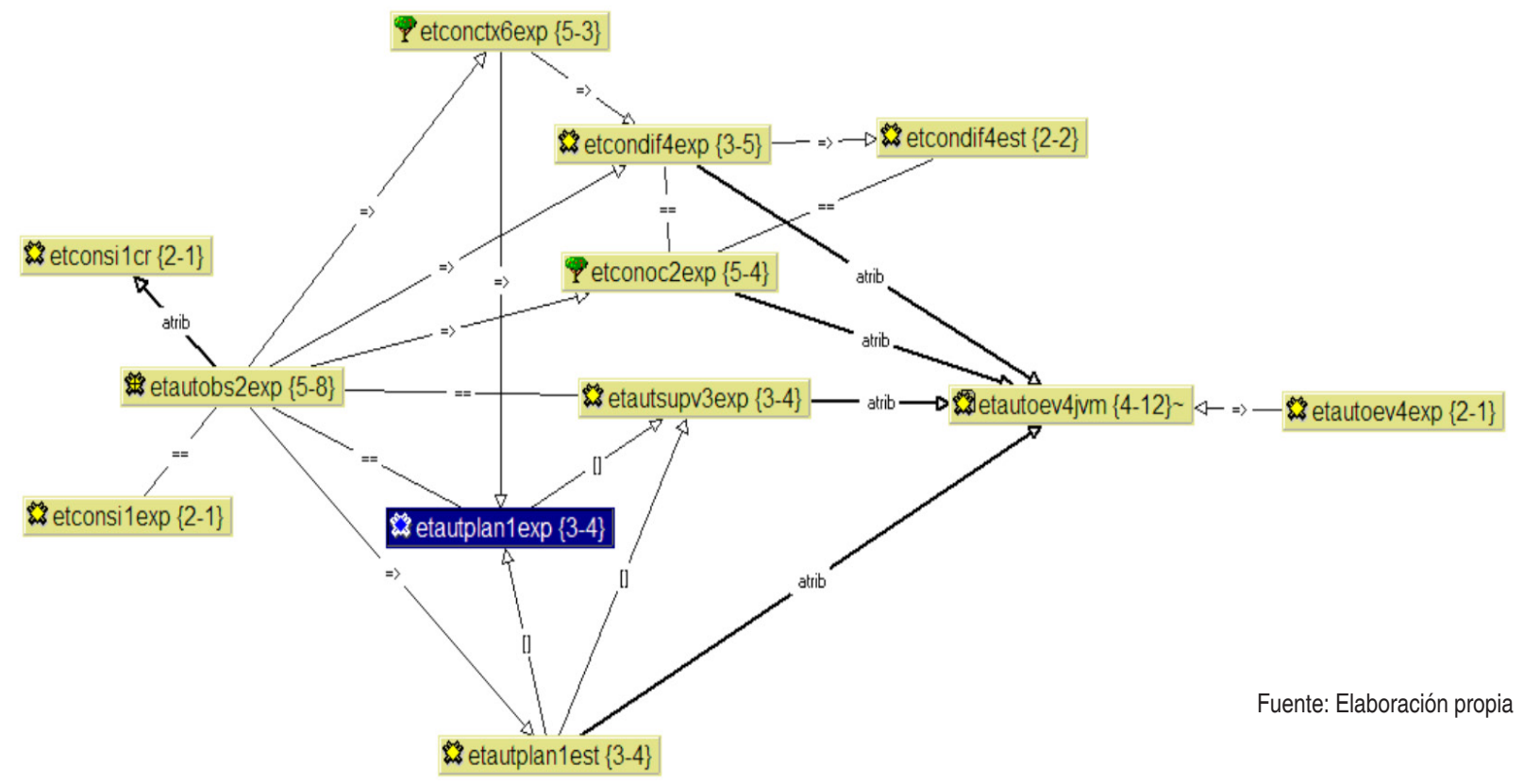



FAMILIA
CODIGO
SUJETOS
CITAS

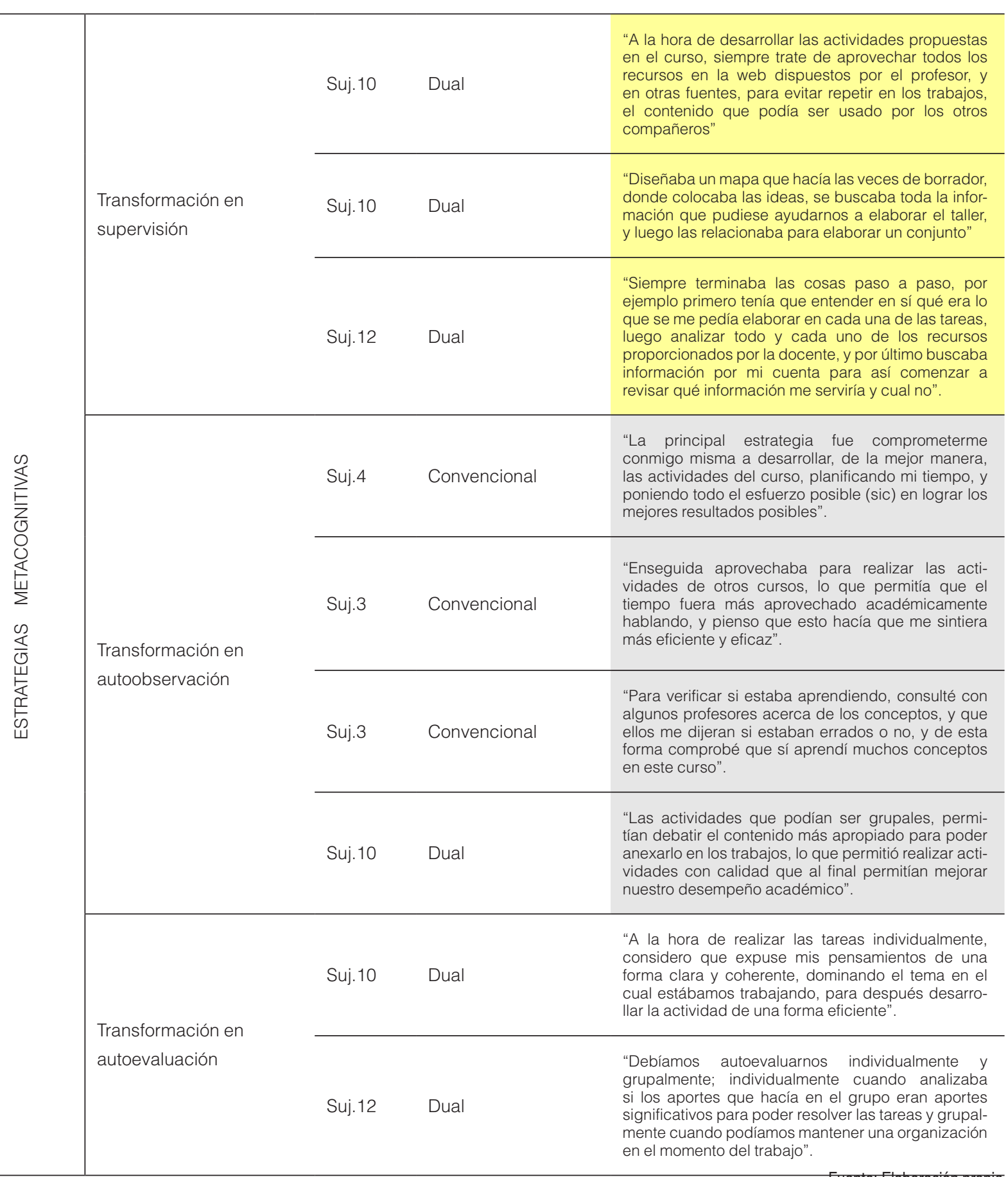




\section{CONCLUSIONES: EL NUEVO LENGUAJE DEL DOCENTE Y LAS NUEVAS REPRESENTACIONES DEL ESTUDIANTE}

La intervención metacognitiva a través de programas de índole educativa diseñados e implementados por el profesorado contribuye a la regulación de la enseñanza y a la autorregulación del aprendizaje en estudiantes universitarios; ello ocurre porque el modelamiento dado desde el lenguaje del profesor, la instrucción y la persuasión metacognitiva, se convierten gradualmente en lenguaje del pensamiento de los estudiantes, indistintamente si están en entornos virtuales, duales o convencionales; ello se puede evidenciar en la creciente expresión de formas de autointerrogación.

En un entorno virtual orientado al desarrollo de actividades de representación, estrategias de aprendizaje cognitivas y metacognitivas, como mínimo el educador debe evidenciar los siguientes rasgos o indicadores en su actuación:

- El profesor activa los conocimientos previos, mediante preguntas e invita a conectar nuevos conocimientos.

- El profesor gestiona recursos y contenidos para configurar experiencias mediadas de aprendizaje situado.

- $\quad$ El profesor promueve en los estudiantes, la realización de mapas e hipertextos para representar relaciones y significados.

- $\quad$ El profesor idea y aplica estrategias para mantenerse informado sobre los progresos de sus estudiantes y sus necesidades de apoyo.

- $\quad$ El profesor aplica formas de evaluación dinámica mediada.

- El profesor propone modelos y situaciones problemáticas invitando a la formulación creativa y heurística de procesos de solución.

- El profesor hace preguntas acerca de las estrategias de aprender; enseña a interrogar y a interrogarse.

- El profesor modela la autointerrogación prospectiva, sincrónica y retrospectiva proponiendo actividades que favorecen en los estudiantes un alto grado de auto-observación, planificación, reflexión evaluación y control de su proceso de aprendizaje.

De este modo, lo que se inicie como estrategias de enseñanza, será apropiado por los estudiantes como formas estratégicas de auto-observación, y de asumir su proceso de organización, elaboración y autoevaluación. Ello se asocia con la redescripción representacional, término utilizado por Karmiloff y Smith (1994). La redescripción representacional sería un factor explicativo de los avances en la autorregulación. Al ser conscientes de sí mismos, los alumnos mejoran su percepción de capacidad (Kluwe, 1982) y del autocontrol como organismos autorreguladores que pueden, deliberada y específicamente, alcanzar progresos en sus metas de aprendizaje.

La autorregulación del aprendizaje en los aprendices universitarios se relaciona entonces con un alineamiento entre autonomía percibida y autonomía expresada; y, a su vez, está mediada por una evolución no solo en la comprensión de sí mismos sino en su capacidad para redescribirse, permanentemente ante sí mismos y ante otros. 
Los p-valores por debajo de 0,05 que permitieron el rechazo de la hipótesis nula para los grupos: a) convencional experimental, b) dual experimental, y c) virtual experimental, en algunas de las subescalas, indican la necesidad de indagar a profundidad, planteando un estudio complementario para establecer si se presentarían variaciones significativas en otras variables a través de una intervención repetida o sostenida y con control más riguroso de variables extrañas; por ejemplo realizando estudios sincrónicos en lugar de un estudio por cohortes como el que se realizó.

El comportamiento, las interacciones y los desempeños de cada estudiante en desarrollo de procesos dentro de un ambiente virtual, al ser registrados por la plataforma dejan una evidencia valiosa que se puede analizar y servir como fuente de información sobre sus representaciones, sus estilos de trabajo y relación con los contenidos, su proceso motivacional y la evolución en sus niveles de comprensión. Un profesor ejerciendo la labor de tutoría puede valerse de esta propiedad de los sistemas de apoyo tecnológico para propiciar itinerarios dinámicos y para suscitar la reflexión sobre los dominios conceptuales, actitudinales y procedimentales de manera que el estudiante reconozca sus disposiciones, habilidades y limitaciones, tomando decisiones sobre lo que requiere y sobre la ayuda que necesita para la gestión de nuevos aprendizajes.

La propuesta desarrollada en esta experiencia docente investigativa corrobora la necesidad de que el profesor mediador asuma un protocolo de comunicación metacognitiva que guíe o promueva la autorregulación de los estudiantes. Así como existen protocolos de uso en las plataformas tecnológicas donde el símbolo y los iconos, sus herramientas y servicios sumergen a los significados en ambientes mediatizados, otro tipo de lenguaje es necesario. Entonces es fundamental traducir la instrucción y la pregunta, clásicos recursos de los ambientes didácticos, en términos de un lenguaje del pensamiento que promueva la interiorización. Ello orientará al estudiante a una mirada permanente sobre sí mismo, sobre sus compromisos epistemológicos y, de orden superior, en la construcción de conocimientos y de sus niveles de comprensión al aprender, así como también para promover estas capacidades desde la conciencia y la autorregulación, las cuales están implícitas en sus procesos de control, planificación y ajuste en sus actividades académicas. Así se irá constituyendo un saber-aprender con trascendencia en su habilidad de aprender permanentemente.

Todo lo anterior implica la necesidad de profundizar en la línea de modelamiento computacional del aprendizaje, del pensamiento, de los estados motivacionales y la representación del conocimiento, y de las competencias del estudiante para integrarlos como componentes de sistemas de acción tecnológico y como promotores del aprendizaje automotivado y autorregulado. A partir de las líneas anteriores, se requerirá la configuración sustentada de nuevas definiciones de aprendizaje y enseñanza, de educabilidad y de enseñabilidad, ahora que la generación de nativos digitales protagoniza los ambientes de formación.

En una cultura universitaria del aprendizaje autónomo, urge la adopción de una praxis en la que cada educador se preocupe por evaluarse a sí mismo, por supervisar su propia actividad y no solo planificarla. Esta serie de actitudes y nuevos hábitos que trascienden a nivel institucional, estarían encaminadas también a reconocer cómo usa el docente los recursos tecnológicos, cómo articula estos recursos a sus ambientes de clase, virtuales, mixtos o 
clásicos; qué busca con esa articulación y qué repercusiones reales en términos de ventajas comparativas tiene en el proceso de aprender de los estudiantes a su paso por la universidad. Por ello, dado que las herramientas tecnológicas, aplicadas al aprendizaje, cumplen una doble función de modificación, tanto interna como externa, porque el sujeto que las usa para transformar información y generar representaciones, en esta interacción, resulta transformado en sus percepciones, sus operaciones instrumentales y cognitivas. Se ha encontrado que los estudiantes autorregulados son más selectivos en la gestión y en las búsquedas, consultan más a los maestros, preguntan a sus compañeros y buscan la ayuda de expertos, dado que ellos reconocen con más seguridad, qué necesitan, en qué área y quién les puede ayudar.

Se debe transformar profundamente lo que se hace y cómo se hace en los ambientes de formación, para que la tecnología surta un efecto en el mejoramiento de la calidad de los aprendizajes; $y$, aunque los recursos técnicos, su disposición y administración jueguen un papel importante, se requiere una nueva actitud frente al aprendizaje, no solo por parte de lo que hacen los estudiantes, sino por lo que hacen los profesores. Se necesitan formas más activas, conscientes y autorreguladas en la gestión del trabajo tanto en los estudiantes como en los docentes, claridad en los propósitos de formación, en las intenciones y en las reglas para garantizar la productividad y la reciprocidad: se trata de aprender-aprender (maestros y alumnos aprenden) y de orientar a aprender de manera motivada, independiente y continua, aprovechando el potencial de las herramientas de mediación tecnológica.

La experiencia realizada ha aportado significativamente a la comprensión del sentido de las prácticas docentes universitarias, no solo en el desarrollo del programa de Licenciatura en Informática de la Universidad de Córdoba, sino en otros programas y facultades, pues es un serio referente para refinar las actividades de aprendizaje que se proponen en el contexto de cursos y aulas virtuales que se crean para complementar la labor presencial, y orientar al trabajo autónomo de los estudiantes.

Los hallazgos han sido discutidos en el contexto de la Maestría en Educación SUE Caribe, programa apoyado por el grupo de investigación CYMTED-L, al cual pertenece la autora de este estudio; se espera que estos resultados sigan siendo valorados como construcción útil en una pedagogía de la autonomía por las instituciones de educación superior en Colombia para que asuman un rol de guía orientadora en los procesos de diseño curricular, y para la formulación de propuestas de innovación, intervención y modelos de diseño de ambientes universitarios de aprendizaje, mediado, y orientados, explícitamente, a la formación en la autonomía. 


\section{Referencias Bibliográficas}

1. Aebli, H. (1991). Factores de la enseñanza que favorecen el aprendizaje autónomo. Madrid: Narcea.

2. Aliste, F.C. (2007). Modelo de comunicación para la enseñanza a distancia. Análisis experimental de una plataforma e-learning. Tesis doctoral. Barcelona: Universidad Autónoma de Barcelona.

3. Azevedo, R. \& Moos, D. C., (2008). Monitoring, planning, and self-efficacy during learning with hypermedia: the impact of conceptual scaffolds. Computers in Human Behavior, 24(4), 1686-1706.

4. Bartolomé P. A. (2004). Blended learning. Conceptos básicos. Pixel Bit. Revista de Medios y Educación 23, 7-20. Sevilla: Universidad de Sevilla. Disponible en http://redalyc.uaemex.mx/ redalyc/pdf/368/36802301.pdf

5. Bausela Herreras, E. (2006). Instrumentos de evaluación, análisis e investigación de la metacognición en relación con la expresión escrita. Lenguaje y textos (10),2 23-24,31-46.

6. Bravo, J.L., Sánchez, J., \& Farjas, M. (2005). El uso de sistemas de b-learning en la enseñanza universitaria. Disponible en http://www.ice.upm.es/wps/jlbr/Documentacion/Uso_bLearComu.pdf

7. Boekaerts, M., \& Niemivirta, M. (2000). Self-regulated learning: finding a balance between learning goals and ego-protective goals. En: M. Boekaerts., P. Pintrich y M. Zeidner (eds). Handbook of Self-Regulation (pp. 417 - 449) California: Academic Press.

8. Pintrich, S ., \& Zeidner, M. (2000). (Eds). Handbook of self-regulation. San Diego, California: Academic Press.

9. Buenfil, R.N. (2008). Análisis de discurso y educación. Recuperado el 12 de noviembre de 2008 desde http://www.uv.mx/uvi/blog/wp-content/uploads/2008/10/unida3_2buenfil.doc

10. Cabero, J., Llorente, M., Cebrián, M., Ruano, I., et al. (2010). Usos del e-learning en las Universidades Andaluzas: estado de la situación y análisis de buenas prácticas. Resumen ejecutivo. Grupo de investigación Didáctica. Universidad de Sevilla: Publidisa S.A.

11. Calés, J.M. (2007). Enseñanza virtual: el modelo de la UNED. Disponible en http://www. rediris.es/difusion/publicaciones/boletin/54-55/ponencia15.html

12. Castañeda, F.S. (2006). Evaluación del aprendizaje en el nivel universitario. Elaboración de exámenes y reactivos objetivos. México: UNAM.

13. Coll, C. (2005). Psicología de la educación y prácticas educativas mediadas por las tecnologías de la información y la comunicación. Una mirada constructivista. Recuperado el 13 de noviembre de 2007 desde http://portal.iteso.mx/portal/page/portal/Sinectica/Historico/Numeros_ anteriores05/025/25\%20Cesar\%20Coll-Separata.pdf. 
14. Coll, C. y Monereo, C. (Comp.). (2008). Psicología de la Educación Virtual. Aprender y enseñar con las tecnologías de la información y la comunicación. Madrid: Morata.Conferencia Regional de Educación Superior, CRES. (2008). Disponible en http://www.universia.net.co/cres2008/destacado/conclusiones-de-la-cres-2008.html.2008.html

15. Copertari, S. \& Trottini, A. M.(2006). Educación virtual y procesos metacognitivos. Un nuevo paradigma de educación a distancia. Ponencia en XIII Congreso de Informática en la Educación.

16. Deed, C. (2009). Strategic Questions: A means of building metacognitive language. International Journal of Teaching and Learning in Higher Education, 20, (3), 481-487. Disponible en http://www.isetl.org/ijtlhe/pdf/JTLHE448.pdf.

17. De la Fuente y Justicia. (2007). Efectos de la utilización de herramientas on-line en la mejora de la regulación del proceso de enseñanza aprendizaje. Revista electrónica de investigación psicoeducativa (13),5, 757-782.

18. De La Torre E., A. (2009). Nuevos perfiles en el alumnado: la creatividad en nativos digitales competentes y expertos rutinarios. En: Cultura digital y prácticas creativas en educación [monográfico en línea]. Revista de Universidad y Sociedad del Conocimiento (RUSC), 1, p.6. UUOC.

19. Donolo, D., Chiecher, A. y Rinaudo, M.C. (2008). Manejo del tiempo y el ambiente en una experiencia didáctica con instancias presenciales y virtuales. RED. Revista de Educación a Distancia, n. ${ }^{\circ}$ 20. Consultado el 13 de enero de 2009 en http://www.um.es/ead/red/20.

20. Duart M., J. \& Sangrà, A. (2000). Aprender en la virtualidad. Barcelona: Gedisa.

21. Duart M., J. \& Lupiáñez, F. (2005). E-strategias en la introducción y uso de las TIC en la universidad. En: Las TIC en la universidad: estrategia y transformación institucional. Revista de Universidad y Sociedad del Conocimiento, RUSC, 2 (1). Disponible en http://www.uoc.edu/rusc/ $\mathrm{dt} / \mathrm{esp} / \mathrm{duart0405}$.pdf.

22. Facundo, Ángel H. (2004). La virtualización desde la perspectiva de la modernización de la educación superior: consideraciones pedagógicas. Revista Universidad y Sociedad del Conocimiento-RUSC. UOC.(1)

23. Fandos, M., Henríquez, P., Gisbert, M. (2000). El diseño de una asignatura virtual para un proyecto interuniversitario. En: Rodríguez, J. (coord.). II Jornadas Multimedia Educativo. Barcelona: Universitat de Barcelona.

24. Feuerstein, R. \& Klein, P. S. Tannenbaum A.J. (1994). Mediated Learning Experience (MLE): Theoretical, psychosocial and learning implications. Tel Aviv, Israel: Reund Publishing House Ltd.

25. Flavell, John H. (1993). Desarrollo Cognitivo. Madrid: Visor

26. Flavell, J., Green, F. (1993). Consciousness. Child Development, 64, pp.387-398.

27. Fumero, A. \& Roca, G. (2007). Web 2.0, Fundación Orange España. Disponible en 
28. http://www.fundacionorange.es/areas/25_publicaciones/WEB_DEFCOMPLETO. pdf.

29. González Cabanach, R., Valle, A. et al. (2000). Diferencias en los componentes cognitivo y afectivo-motivacional entre distintos niveles de aprendizaje autorregulado en estudiantes universitarios. Bordón. Revista de pedagogía (52), 4. pp 537-553.

30. González, J., Wagenaar, R., \& Beneitone, P. (2004). Tuning América Latina: un proyecto de las universidades. Revista Iberoamericana de Educación, 35, 151-164.

31. 29. Kaplan, A. (2008). Clarifying Metacognition, Self-Regulation, and Self-Regulated Learning: What's the Purpose? Educational Psychology Review, 20, 477-484.

32. Lanz, M (2006). Self regulated learning: the place of cognition, metacognition and motivation. Revista Estudios Pedagógicos, 32(2). Valdivia: Falta editorial.

33. Lara, R., Sabogal, L. \& Palmezano, R. (2006). Validación del inventario de Estilos de aprendizaje y de Orientación Motivacional (EDAOM) en estudiantes universitarios. Impreso Universitario. Universidad del Magdalena. Colombia.

34. Leal, D. (2008). Iniciativa colombiana de objetos de aprendizaje: situación actual y potencial para el futuro. Apertura, 8 (8), 76-85. Disponible en http://redalyc.uaemex.mx/redalyc/ pdf/688/68811215006.pdf.

35. Lezama, O. (2004). El Programa Universidad Virtual como instrumento de ampliación de cobertura educativa y modernización de la Universidad Nacional de Colombia. Disponible en http://www.unal.edu.co/noticias/claustros/colegiaturas/Universidad/virtual_2004.doc.

36. Martínez, F. y Prendes, M. (2007). Nuevas Tecnologías y Educación, pp 93-145, 215. Madrid: Pearson. Prentice Hall.

37. Martínez, J.R. (2004). Concepción de aprendizaje, metacognición y cambio conceptual en estudiantes universitarios de Psicología. Tesis doctoral. Universidad de Barcelona. Disponible en http://www.tesisenxarxa.net/TESIS UB/AVAILABLE/_TDX-1006104-091520/Tesis_final.pdf.

38. McVay L, M. (2004). Learning Online: A Guide to Success in the Virtual Classroom. New York: Routledge Falmer.

39. Mithaug, D. E. \& Deirdre K., M. et al.(2003). Self-Determined Learning Theory Construction, Verification and Evaluation. New Jersey: Lawrence Erlbaum Associates.

40. Montenegro, I. (2004). Influencia de Preguntas Cognitivas y Metacognitivas en el proceso de aprendizaje en ciencias con el apoyo de un sistema inteligente. Tesis doctoral. Universidad Pedagógica Nacional , Colombia.

41. Moos, D. C., \& Azevedo, R. (2008). Self-regulated learning with hypermedia: the role of prior domain knowledge. Contemporary Educational Psychology, 33(2), 270-298.

42. Pérez, E., Delgado, M. (2006). Self-efficacy for Study Inventory. Development and initial validation. Universidad Nacional de Córdoba. Argentina. Recuperado el 3 abril 2008 en http://www. 
des.emory.edu/mfp/PerezAutoregulacion.pdf. Plan Nacional de Tecnologías de la Información y las Comunicaciones-2008-2019. Bogotá: Ministerio de Educación Nacional de Colombia.

43. Pedraza, M. (2006). Los entornos virtuales de enseñanza-aprendizaje: propuesta pedagógica. Recuperado el 23 de abril de 2007 desde http://site.ebrary.com/lib/univgranada/ Doc?id=10119227\&ppg $=10$.

44. Rondón, M. (2007). Modelos Virtuales en las Instituciones de Educación Superior Colombianas. Informe Viceministerio de Educación Superior. Disponible en http://www.colombiaaprende.edu.co/html/mediateca/1607/articles-126604_archivo.pdficeministerio.

45. Salovaara, H. (2005). An exploration of students' strategy use in inquiry-based computersupported collaborative learning. Journal of Computer Assisted Learning, 21(1), 39-52.

46. Sánchez, A. y Vovides, Y. (2007). Integration of metacognitive skills in the design of learning objects. Computers in Human Behavior. Amsterdam: Elsevier Science Publishers.

47. Schunk, D. H. (2008). Metacognition, Self-Regulation, and Self-Regulated Learning: Research Recommendations. Educational Psychology Review, 20, 463-467.

48. Sierra, P. I. (2008). Docencia interactiva en entornos virtuales. Escenarios y posibilidades, pp. 63-68. Universidad de Córdoba-Colombia. Montería: Alpha comunicación estratégica.

49. Sierra, P. I. (2009). Función didáctica y dimensión social cognitiva en el diseño de entornos virtuales: implicaciones para la evaluación dinámica y el desarrollo del aprendizaje autónomo. Ponencia en: Primer Congreso Internacional de Ambientes Virtuales de Aprendizaje Adaptativos y Accesibles. Montería

50. Winters I., F. \& Greene, J. A. \& Costich, M .(2008). Self-Regulation of Learning within Computer-based Learning Environments: A Critical Analysis. Educational Psychology Review 20,429-444.

51. Zapata, D. (2004). Contextualización de la enseñanza virtual en la educación superior. Bogotá: ICFES.

52. Zea, C. et. al. (2005). Hacia un modelo de formación continuada de docentes de educación superior en el uso pedagógico de las tecnologías de información y comunicación. Bogotá: Colciencias.

53. Zimmerman, B. J. y Dale H., S. (2001). Self-Regulated Learning and Academic Achievement: Theoretical Perspectives. Mahwah, New Jersey: Lawrence Erlbaum Associates. Azevedo, R. \& Moos, D. C., (2008). Monitoring, planning, and self-efficacy during learning with hypermedia: the impact of conceptual scaffolds. Computers in Human Behavior, 24(4), 1686-1706. 